\title{
Change of Electrophysical Properties of Nanocrystalline SiC Films by Laser Treatment at Applied Electric Field
}

\author{
A. V. Semenov, ${ }^{1}$ V. M. Puzikov, ${ }^{1}$ P. V. Mateichenko, ${ }^{1}$ and V. Romano ${ }^{2}$ \\ ${ }^{1}$ Institute for Single Crystals, National Academy of Sciences of Ukraine, Kharkiv 61001, Ukraine \\ ${ }^{2}$ Bern University of Applied Science, 3400 Burgdorf, Switzerland
}

Correspondence should be addressed to A. V. Semenov; semenov@isc.kharkov.ua

Received 6 June 2013; Accepted 3 July 2013

Academic Editors: I. L. Li and F. Sansoz

Copyright (C) 2013 A. V. Semenov et al. This is an open access article distributed under the Creative Commons Attribution License, which permits unrestricted use, distribution, and reproduction in any medium, provided the original work is properly cited.

\begin{abstract}
Studied are peculiarities of the changes in the structure and electrophysical properties of nanocrystalline silicon carbide films of 3C$\mathrm{SiC}$ polytype subjected to the action of picosecond laser pulses with $\lambda=355 \mathrm{~nm}$ and a pulse power up to $1.5 \mathrm{~W}$. It is established that laser processing of the films with an energy density of $3 \times 10^{-2}-30 \mathrm{~J} / \mathrm{cm}^{2}$ leads to ablation without decomposition of SiC. During the laser processing the electrical resistance of the films rises due to diminution of the film thickness. While measuring the PL properties of nc-SiC films under the influence of the applied electric field with intensity $3 \times 10^{3} \mathrm{~V} / \mathrm{cm}$, the effect of a single triple enhancement of the luminescence maximum is revealed. Repeated PL measurements at the same area of the film under the applied electric field with the intensity ranging from 0 to $1 \times 10^{4} \mathrm{~V} / \mathrm{cm}$ show that the PL emission intensity diminishes at the short-wavelength boundary of the maximum and rises at the long-wavelength boundary. Thereat the spectral position of the PL maximum remains unchanged.
\end{abstract}

\section{Introduction}

Nanocrystalline silicon carbide (nc-SiC) films are promising for micro- and nanomechanical systems used for registration, measurement, and analysis of different external factors, including most severe ones [1]. High resistance of $\mathrm{SiC}$ atomic structure to electromagnetic fields makes it possible to analyze the electronic properties of thin $\mathrm{nc}-\mathrm{SiC}$ layers using high-power ultrashort laser pulses for excitation of the electronic subsystem. At the same time, irradiation of thin $\mathrm{SiC}$ layers by powerful femto- and picosecond laser pulses gives rise to the problems connected with possible changes in the structure and phase composition of the material under the action of high peak intensity electromagnetic pulses. The latter may stimulate photochemical reactions [2], surface local melting [3], and ablation [4]. In particular, it has been established in $[5,6]$, that such a treatment results in decomposition of crystalline silicon carbide into carbon and silicon phases, which cause the rise of the electric conductivity and the appearance of fused silicon in the treatment channel, respectively. At the same time, data on the use of high-power ultrashort laser pulses for the modification of the structure of nanocrystalline silicon carbide and investigation of its electronic are practically absent.

Therefore, the aim of the present work was to study of the possibility to apply high-power picosecond laser irradiation for modification of the electrophysical properties of nc$\mathrm{SiC}$ films and measurement of their photoluminescent (PL) properties under the conditions of simultaneous action of picosecond laser radiation and electric field $(E)$.

The latter is connected with the fact that nc-SiC films in MEMS facilities are conventionally affected by electric fields of different intensity, and it is greatly significant to establish their influence on the processes of excitation and charge transfer. The values of irradiation power which stimulated the onset of structural changes in $\mathrm{nc}-\mathrm{SiC}$ films were determined from the changes in the electrical resistance $(R)$ of the films under the action of picosecond laser UV radiation. The said 


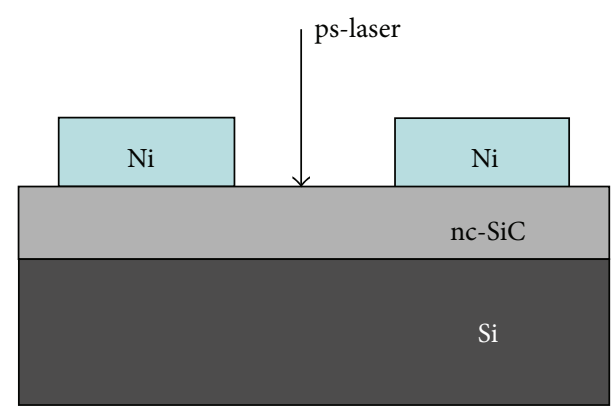

FIgUre 1: Cross-sectional scheme of the sample of nc-SiC films for measurement of changes in the electrical resistance under laser irradiation.

measurements were accompanied with the analysis of the change of the film surface morphology determined by means of a scanning electron microscope.

\section{Experimental}

The study was performed on nc-SiC films obtained by the method of direct ion deposition $[7,8]$ on crystalline sapphire and silicon substrates. The films deposited on the substrates at a temperature of $1000^{\circ} \mathrm{C}$ had a closely packed structure based on nanocrystals of the cubic polytype $3 \mathrm{C}-\mathrm{SiC}$ with an average nanocrystal size of $10-15 \mathrm{~nm}$ and a thickness ranging between 300 and $800 \mathrm{~nm}$. Using the method of vacuum evaporation there were formed linear nickel contacts on the film surface with copper conductors joined by indium solder. The distance between the contacts with a width of $2-3 \mathrm{~mm}$ was $0.6-2 \mathrm{~mm}$. The frontal and cross-sectional views of the samples are shown in Figure 1.

The films were irradiated using a picosecond laser system DUETTO4, the radiation wavelength and pulse duration were $355 \mathrm{~nm}(3.49 \mathrm{eV})$ and $10 \mathrm{ps}$, respectively, and the average pulse power was varied from 015 to $1.5 \mathrm{~W}$. The resistance of the films was modified by radiation scanning of the interelectrode space, at a beam scanning velocity of $3 \times$ $10^{5} \mu \mathrm{m} / \mathrm{s}$, with the diameter of the focal spot on the samples being $10 \mu \mathrm{m}$. There was no spacing between the adjacent scanning bands. The pulse energy density varied in $3 \times$ $10^{-2}-30 \mathrm{~J} / \mathrm{cm}^{2}$ range. Photoluminescence was excited in the films by laser radiation with $\lambda_{\text {exc }}=355 \mathrm{~nm}(3.49 \mathrm{eV})$ using the system DUETTO4, and the radiation pulse duration was $10 \mathrm{ps}$, the average radiation power varied in $0.15-1.5 \mathrm{w}$ range, the diameter of laser excitation spot on the film was $500 \mu \mathrm{m}$. The photoluminescence spectra were measured at room temperature in the interelectrode space, using a fiber optical spectrometer USB-4000 with input lens that focused on the film on the excitation spot. Electric field in the spacing between the electrodes was created in $0-1 \times 10^{4} \mathrm{~V} / \mathrm{cm}$ range using stabilizing DC power sources Delta Elektronika E0300$0.1(0-100 \mathrm{~mA}, 0-600 \mathrm{~V})$. The electrical conductivity was measured by a Multimeter/Supply Agilent U 3606 A, with an output voltage of 2-3 V. The morphology and composition of the film surface were analyzed using a scanning electron microscope JSM-6390 LV.

\section{Change of Electrical Conductivity in nc-SiC Films by Picosecond Laser Processing}

To modify the electrical conductivity of nc-SiC films by laser processing of the space between the electrodes was continuously scanning by laser beam with a diameter of $10 \mu \mathrm{m}$ along the normal to the end of the electrodes. The scanning area completely overlapped the electric circuit between the electrodes. Figure 2 presents the electron microscopic images of successive changes in the morphology of nc-SiC after laser treatment with different pulse energy fluences. Each image was obtained after 10 scans by laser beam with a preset energy density. The laser pulse fluencies were successively changed with in $4 \times 10^{-4}-3 \times 10^{-1} \mathrm{~J} / \mathrm{cm}^{2}$ range. The microphotographs show ordered strips forming a grid with pits of a diameter of about $5 \mu \mathrm{m}$ located in the nodes. These pits are due to ablation of the film by the central part of the laser beam with a Gaussian distribution of the intensity. As seen from the presented scale, the lattice parameter was $10 \mu \mathrm{m}$ that proved to the fact that the laser beam with a diameter of $10 \mu \mathrm{m}$ completely covered the film at scanning. In other words, though the completely surface was subjected to laser processing, the removal of the material was more intense under the action of the central part of the beam with a diameter of $5 \mu \mathrm{m}$, due to a Gaussian distribution of the intensity in the beam. The microphotographs show that the dependence of the changes in the morphology on the number of scans becomes stronger as the pulse energy density increases.

The morphological changes are characterized by the absence of visible traces of melting of the material, though the laser radiation wavelength $(355 \mathrm{~nm})$ is within the spectral region of fundamental absorption of nc-SiC films [8]. Moreover, such traces are also absent in the whole range of the energy pulse densities. So, the removal of nc-SiC may be caused by the ablation at radiation energy densities of 4.3 $\times 10^{-4}-30.1 \times 10^{-2} \mathrm{~J} / \mathrm{cm}^{2}$. As noted in $[5,9]$, laser treatment of single-crystalline $\mathrm{SiC}$ films with close radiation energy parameters leads to decomposition of silicon carbide into the carbon and silicon phases and to melting of silicon. Thus, one may conclude that at 10 ps pulse duration, an energy density of $4 \times 10^{-4} \mathrm{~J} / \mathrm{cm}^{2}$ is sufficient for the ablation of $\mathrm{nc}-\mathrm{SiC}$ with an average nanocrystal size of $10-15 \mathrm{~nm}$. The electrical resistance $R$ of the film is more sensitive to the changes in the structure and phase composition under laser irradiation. The changes of this parameter are shown in Figures 3(a) and $3(\mathrm{~b})$. The initial resistance in the space between the contacts ranged between $10^{4}$ and $10^{5} \mathrm{Ohm}$ depending on the film thickness and the geometry of the contacts. Figures $3(a)$ and 3 (b) present the dependencies of the changes in $R$ for $\mathrm{nc}-\mathrm{SiC}$ films on the treatment by laser beam with different intensity, for the samples treated at a step of 1 scan (a) and 10 scans (b). The energy density was raised each 10 scans. The common feature of both dependences is the rise of the resistance at any laser treatment intensities. This allows to make the following two conclusions. Firstly, the ablation of the material occurs without the decomposition of $\mathrm{SiC}$ into carbon and silicon. Secondly, the rise of the resistance is caused by the diminution of the cross-section of the film due to the 

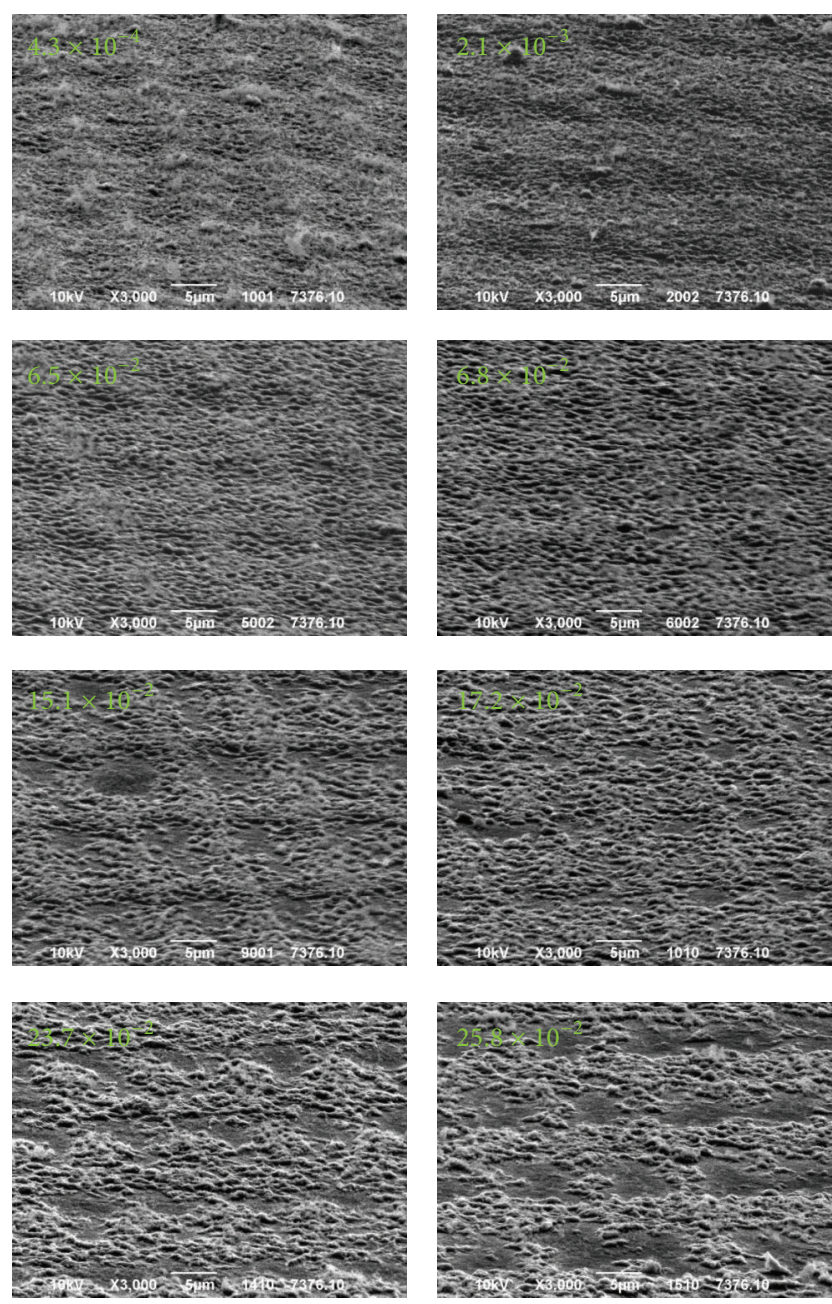
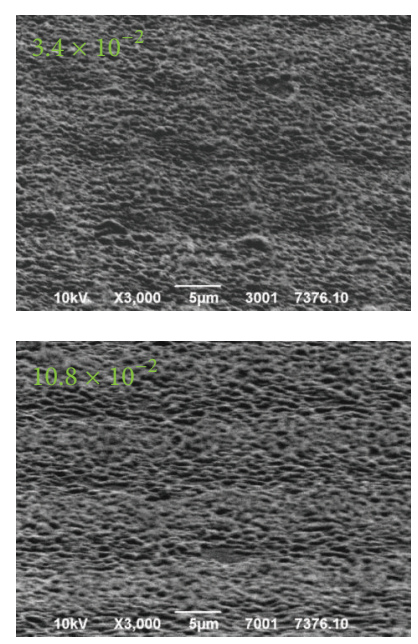
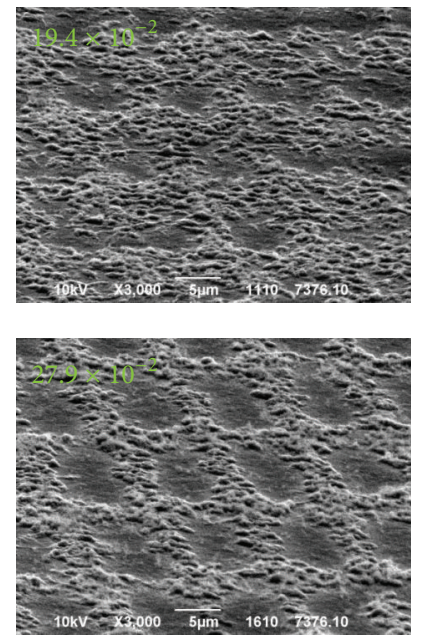
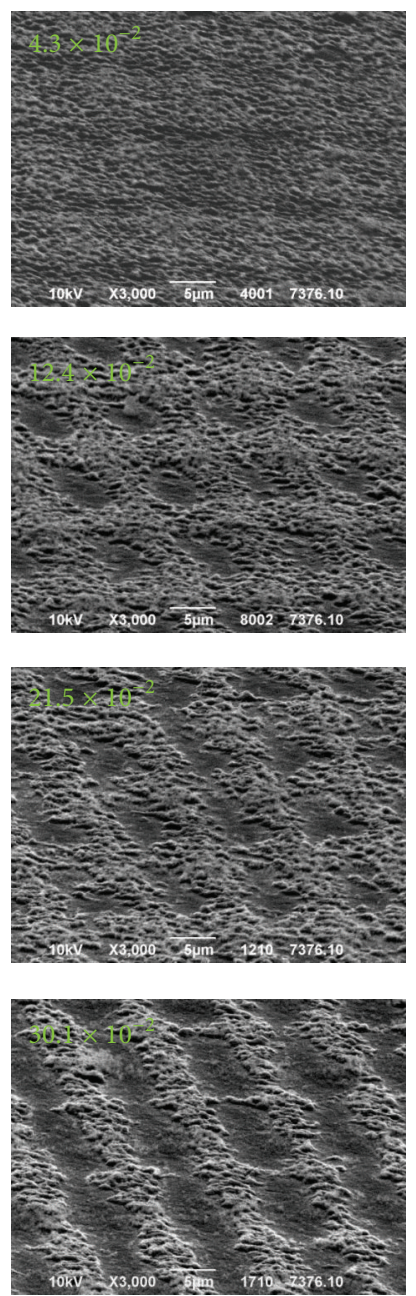

FIGURE 2: Scanning electron microscopic images of successive changes in the morphology of nc-SiC film after laser processing with different energy pulse density, $\mathrm{J} / \mathrm{cm}^{2}: 1: 4.3 \times 10^{-4}, 2: 2.1 \times 10^{-3}, 3: 4.3 \times 10^{-2}, 4: 4.3 \times 10^{-2}, 5: 6.5 \times 10^{-2}, 6: 8.4 \times 10^{-2}, 7: 10.8 \times 10^{-2}, 8: 12.4 \times 10^{-2}, 9: 15.1$ $\times 10^{-2}, 10: 17.2 \times 10^{-2}, 11: 19.4 \times 10^{-2}, 12: 21.5 \times 10^{-2}, 13: 23.7 \times 10^{-2}, 14: 25.8 \times 10^{-2}, 15: 27.9 \times 10^{-2}$, and $16: 30.1 \times 10^{-2}$.

removal of the material. Such a behavior of nc-SiC under the conditions of high-power ultrashort laser irradiation sharply differs from that of single-crystalline $\mathrm{SiC}$ resistance of which falls under laser irradiation due to the decomposition of silicon carbide into carbon and silicon $[5,9]$. The presented dependences show insignificant rise of the film resistance under the treatment at energy densities of about up to 0.15$0.2 \mathrm{~J} / \mathrm{cm}^{-2}$, whereas the electron microscopic images allow to observe the change of the surface morphology. This may prove to be due to the inhomogeneity of the structure and phase composition in the depth of the films, as well as to the fact that the substrate-adjacent layer of $\mathrm{nc}-\mathrm{SiC}$ is more responsible for the resistance of the film. This is natural for thin films formed by deposited ions with elevated kinetic energy. As seen from both graphs, at pulse energy densities higher than $0.2 \mathrm{~J} / \mathrm{cm}^{-2}$, the resistance sharply rises, and this gives evidence of violation of the film's continuity. According to the electron microscopic images (Figure 2, images 11-16), the removal of the film is followed by the appearance of clean substrate surface. It should be noted that, due to insignificant absorption of sapphire substrate at the working wavelength $\lambda=355 \mathrm{~nm}$, complete ablation of thin $\mathrm{nc}-\mathrm{SiC}$ film occurs without heating or the substrate ablation. After the onset of rapid rise of the resistance at energy densities exceeding $0.2 \mathrm{~J} / \mathrm{cm}^{2}$, one can see the so-called shelves on these dependences. On the curve (3a) such a shelf is less pronounced than on the curve (3b). As seen from the microimages (Figure 2, images 9,10 ), the appearance of the shelves corresponds to the formation of insular morphology of the films. Electrical conduction between the islets occurs via the bridges which connect them. Therefore, the appearance of the shelf on the dependence of the resistance on the intensity of laser ablation testifies that the rate of ablation of the islet materials is characterized by spatial anisotropy. In other words, the ablation intensity in the surface plane is lower than the one in the direction normal to the film surface. This is connected with overdeposition of the material in the film plane. Further rise of the pulse energy density leads to complete disturbance of the film's continuity and of electrical conduction between the islets. So, the resistance becomes too high to be measured 


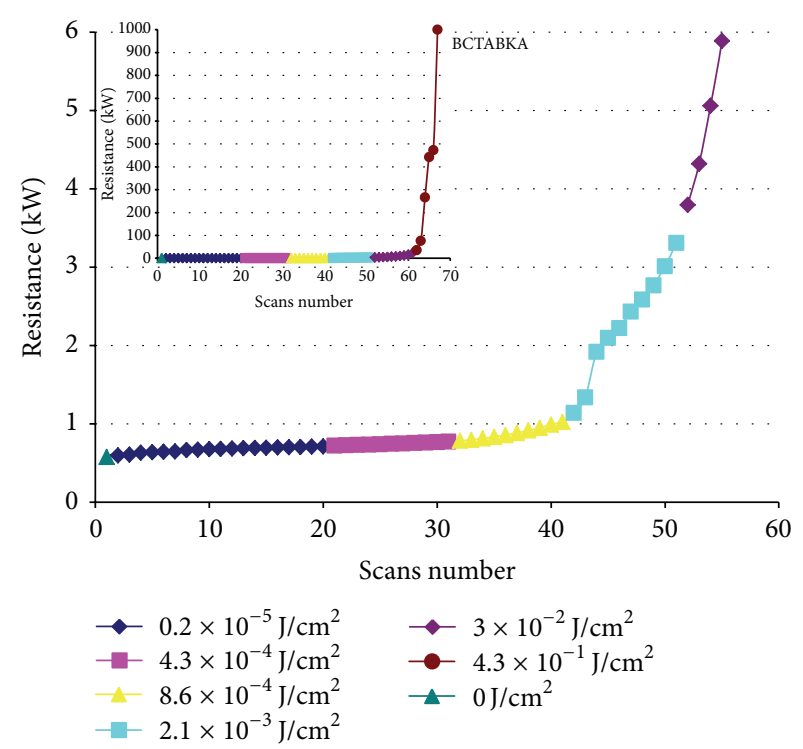

(a)

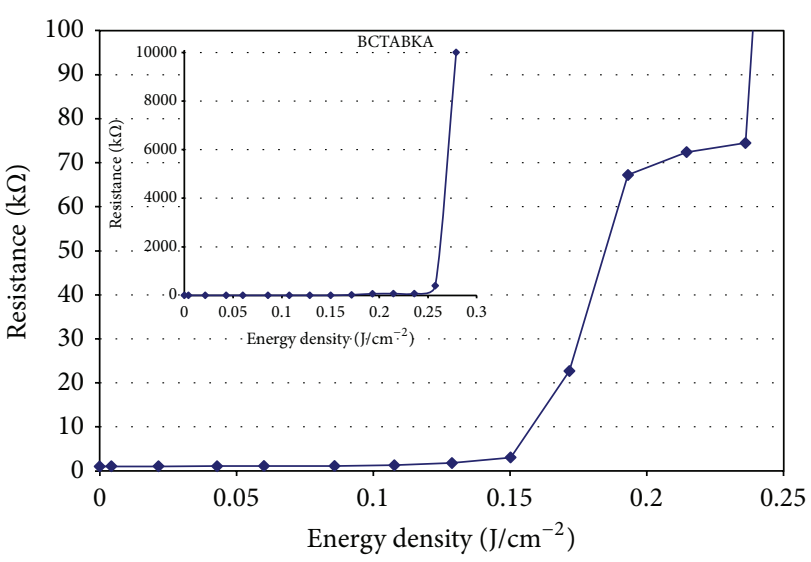

(b)

FIGURE 3: Dependence of the changes in $R$ of nc-SiC on laser beam processing at different intensity. (a) With a step of 1 scan, (b) with a step of 10 scans. In both cases the energy density was increased in each of 10 scans.

by our multimeter. This means that we measure the surface resistance of the used sapphire substrates.

We presented the results of the resistance measurements for $\mathrm{nc}-\mathrm{SiC}$ films subjected to picosecond laser treatment obtained after switching off the laser. However, the resistance measured during laser irradiation was found to be $10-15 \%$ lower. While measuring $R$ by the multimeter, the electric field intensity in the space between the electrodes was $\sim 10 \mathrm{~V} / \mathrm{cm}$. Naturally, the additional conductivity in the films was caused by photoexcitation of localized charge carriers in the conduction channels. After switching off the laser complete relaxation of photocarriers occurred in several minutes.

\section{Effect of Applied Electric Fields on Photoluminescence in nc-SiC Films}

Photoluminescence of nc-SiC films with applied electric field was excited by laser radiation with an energy density by two orders less $\left(<2 \times 10^{-5} \mathrm{~J} / \mathrm{cm}^{2}\right)$ than the one leading to the ablation. The PL spectra were measured at quasi-continuous laser excitation of the films at varying the electric field intensity in $0-1 \times 10^{4} \mathrm{~V} / \mathrm{cm}$ range with a discreteness of $5 \times$ $10^{2} \mathrm{~V} / \mathrm{cm}$. Presented in Figure 4(a) are the PL spectra for the nc-SiC film without electric field applied (1) and with the field applied for the first time (2). The excitation energy density was equal to $2.4 \times 10^{-5} \mathrm{~J} / \mathrm{cm}^{2}, E=3 \times 10^{3} \mathrm{~V} / \mathrm{cm}$. Figure $4(\mathrm{~b})$ contains the $\mathrm{Pl}$ spectrum at the same point with elevated excitation energy density $\left(4.8 \times 10^{-5} \mathrm{~J} / \mathrm{cm}^{2}\right), E=3 \times 10^{3} \mathrm{~V} / \mathrm{cm}$. This spectrum was obtained in $20 \mathrm{~min}$. of the measurement of the first spectrum. As is seen, in the absence of electric field, both spectra contain a wide nonsymmetrical band with a maximum in the region of $510-520 \mathrm{~nm}$ characteristic of
PL of nc-SiC film of the cubic polytype [10]. This band is difficult for analysis, due to widening of the maximum of the PL band and to its location close to the exciting laser radiation wavelength $(355 \mathrm{~nm})$. However, it may be concluded that the main contribution to the emission is made by the radiative indirect interband transitions $\Gamma 1_{5 v}-X_{1 c}$ in cubic silicon carbide $(517 \mathrm{~nm})$ [11].

The observed widening of this band seems to be due to the contribution of radiative transitions involving the states localized near the edges of the forbidden band. Such states may generate impurity and structure defects in the layer of $\mathrm{nc}-\mathrm{SiC}$ formed at deposition of ions with an energy exceeding $100 \mathrm{eV}$. At the application of external electric field to the nc$\mathrm{SiC}$ film we observed two different phenomena. The one of them was a sharp increase of the emission intensity of the main PL band observed immediately after the first switching on. As seen from Figure 4(a), after switching on the electric field with an intensity of $4 \times 10^{3} \mathrm{~V} / \mathrm{cm}$ for the first time, the intensity of the signal increased by approximately 3 times. However, such an effect was not stationary and remained for about 2 minutes. Then the initial signal value was restored, and one could observe slight changes in the spectrum to be described lower. The attempts to achieve another sharp rise of the luminescence intensity for the same sample which lasted approximately an hour were unsuccessful. Shown in Figure 4(b) is the change of the PL spectrum measured at the same point of the film, with elevated pulse energy density and the field switched on. One can see that PL signal enhancement was absent; that is, this effect was single. Similar dependence of PL on the applied electric field earlier observed for ZnSbased microcrystalline luminophores is named after the authors, Gudden-Pole effect (GPE) [12]. This phenomenon revealed about 100 years ago has not been unambiguously described in the literature so far. Some authors explain GPE 


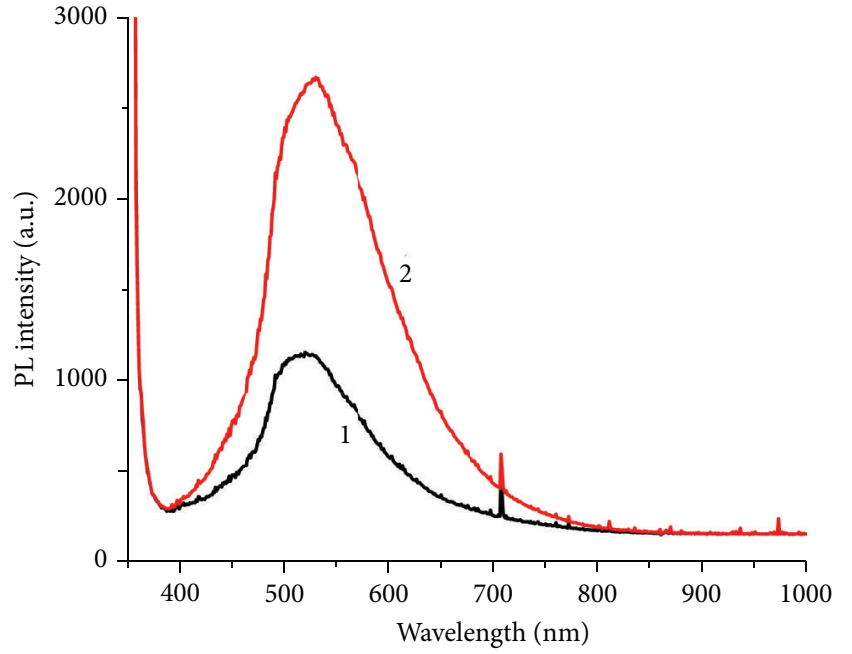

(a)

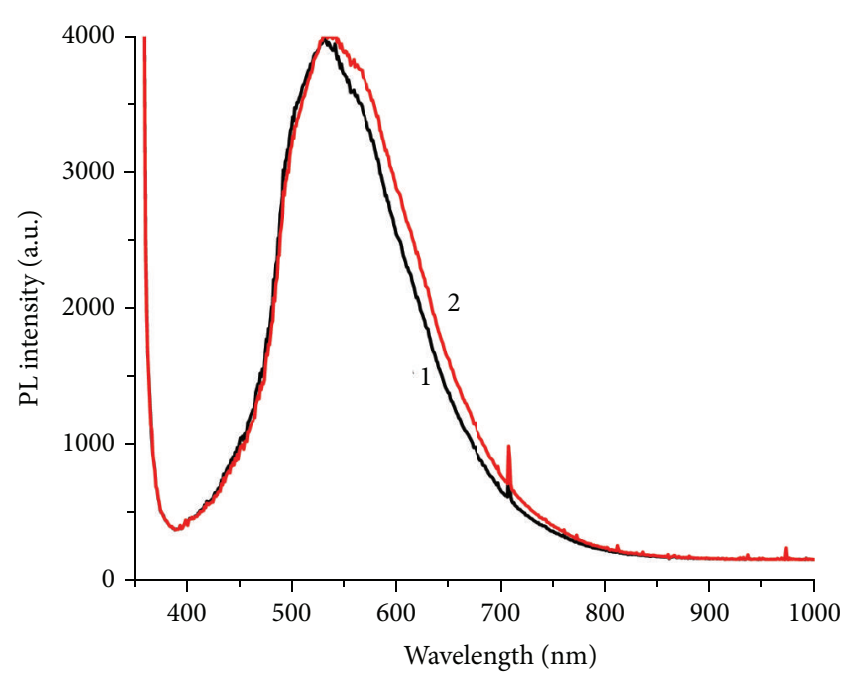

(b)

FIGURE 4: PL spectra in nc-SiC film depending on the intensity $E$ of electric field intensity applied for the first time (a) excitation energy density $=24 \mathrm{~mJ} / \mathrm{cm}^{2}, E=4 \times 10^{3} \mathrm{~V} / \mathrm{cm}$, (b) PL spectra for the same film area with elevated energy density $\left(48 \mathrm{~mJ} / \mathrm{cm}^{2}\right)$ and repeated application of the field $\left(E=4 \times 10^{3} \mathrm{~V} / \mathrm{cm}\right)$.

by liberation of electrons from shallow traps under the action of electric field followed by radiative recombination on the emission centers. The main mechanism of field delocalization of electrons in solids at GPE is electric charge tunneling stimulated by photons [13] The influence of electric field on $\mathrm{PL}$ of the films is characterized by the fact that the electric excitation may have a long-term action (e.g., for several hours) [14]. This means that at first excessive electrons excited by electric field increase the luminescence light yield and then they are captured by deep traps for a long period of time. The localization time depends on the depth of the trap and the probability of its ionization. Therefore, in our experiments, we did not observe repeated enhancement of PL at switching on the field for the same sample at least for an hour.

The other phenomenon revealed in our experiments was the following. After the first enhancement of PL and restoration of the initial signal value we observed a slight spectral shift of the boundaries of the PL maximum with the rise of the applied electric field intensity. Presented in Figure 5 is the dependence of the PL spectra on the applied field measured after the GPE in another interelectrode space of nc-SiC film. As seen from Figure 5 (and Figure 4(b)), at the increase of the electric field intensity in $0-1 \times 10^{4} \mathrm{~V} / \mathrm{cm}$ range, the UV boundary of the maximum becomes lower, whereas the long-wavelength boundary lifts approximately by $25 \%$. It is difficult to estimate the change of the UV boundary, since the wing of primary excitation superimposes on it. It seems natural to expect that the intensity of the PL maximum at the UV boundary will be lower by the same value. Thereat, the spectral position of the maximum remains unchanged. This testifies to the fact that in $\mathrm{nc}-\mathrm{SiC}$ layer the applied electric field gives rise to redistribution of the occupancy of the traps near the edges of the valence and conduction bands where radiative electron recombination occurs. It may be assumed that the states in the conduction bands are being filled (i.e.,

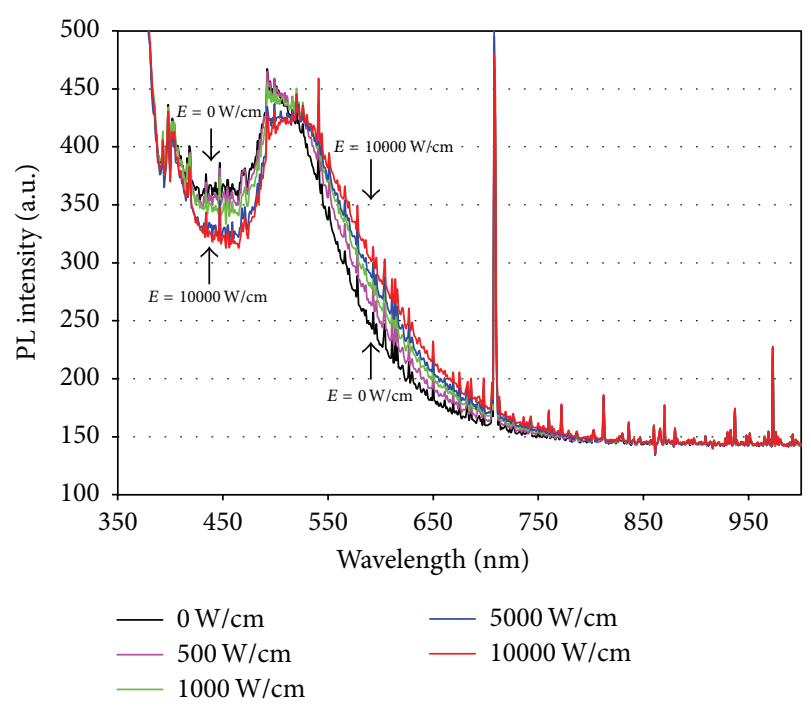

Figure 5: PL spectra in nc-SiC film depending on the intensity of the applied electric field. The excitation energy density $=24 \mathrm{~mJ} / \mathrm{cm}^{2}$. The electric field intensity was raised from 0 up to $1 \times 10^{4} \mathrm{~V} / \mathrm{cm}$ at a step of $5 \times 10^{2} \mathrm{~V} / \mathrm{cm}$.

confirmed by the appearance of current in the film) and do not participate in the radiative transitions with energies exceeding the band gap value $(>2.4 \mathrm{eV})$. Thereat, the localized states below the conduction band are released, and quantity of transitions which emit photons with an energy lower than $2.4 \mathrm{eV}$ increases. Thus, the red boundary of the PL maximum rises.

\section{Conclusion}

Experimentally studied are peculiarities of the changes in the structure and electrophysical properties of nanocrystalline 
silicon carbide films of 3C-SiC polytype subjected to the action of picosecond laser pulses with $\lambda=355$ and a pulse power up to $1.5 \mathrm{wt}$. It is established that laser processing of the films with an energy density of $3 \times 10^{-2}-30 \mathrm{~J} / \mathrm{cm}^{2}$ leads to ablation without decomposition of SiC. During the laser processing the electrical resistance of the films rises due to diminution of the film thickness.

While measuring the PL properties of nc-SiC films under the influence of the applied electric field with $E=3 \cdot 10^{3} \mathrm{~V} / \mathrm{cm}$, the effect of a single triple enhancement of the luminescence maximum corresponding to the interband radiative transition $\Gamma_{15 v}-X_{1 c}$ in cubic silicon carbide is revealed. Repeated PL measurements at the same area of the film under the applied electric field with the intensity ranging from 0 to 1 $\times 10^{4} \mathrm{~V} / \mathrm{cm}$ show that the PL emission intensity diminishes at the short-wavelength boundary of the maximum and rises at the long-wavelength boundary. There at the position of the PL maximum remains unchanged.

\section{Acknowledgments}

The authors are grateful to assistants M. Muralt, R. Augsburger, B. Jaeggi, and J. Boas of Bern University of Applied Science, for their help in the laser experiments. This work was supported by the Swiss National Science Foundation Project IZ73Z0_128088/1.

\section{References}

[1] M. Mehregany and C. A. Zorman, "SiC MEMS: opportunities and challenges for applications in harsh environments," Thin Solid Films, vol. 355, pp. 518-524, 1999.

[2] Y. Shimotsuma, M. Sakakura, S. Kanehira et al., "Threedimensional nanostructuring of transparent materials by the femtosecond laser irradiation," Journal of Laser Micro / Nanoengineering, vol. 1, pp. 181-186, 2006.

[3] S. Y. Chou, Y. Chang, K. H. Weiner, T. W. Sigmon, and J. D. Parsons, "Annealing of implantation damage and redistribution of impurities in SiC using a pulsed excimer laser," Applied Physics Letters, vol. 56, no. 6, pp. 530-532, 1990.

[4] M. D. Perry, B. C. Stuart, P. S. Banks, M. D. Feit, V. Yanovsky, and A. M. Rubenchik, "Ultrashort-pulse laser machining of dielectric materials," Journal of Applied Physics, vol. 85, no. 9, pp. 6803-6810, 1999.

[5] M. Deki, T. Ito, M. Yamamoto et al., "Enhancement of local electrical conductivities in $\mathrm{SiC}$ by femtosecond laser modification," Applied Physics Letters, vol. 98, no. 13, Article ID 133104, 2011.

[6] I. A. Salama, N. R. Quick, and A. Kar, "Laser doping of silicon carbide substrates," Journal of Electronic Materials, vol. 31, no. 3, pp. 200-208, 2002.

[7] A. V. Semenov, V. M. Puzikov, M. V. Dobrotvorskaya, A. G. Fedorov, and A. V. Lopin, "Nanocrystalline SiC films prepared by direct deposition of carbon and silicon ions," Thin Solid Films, vol. 516, no. 10, pp. 2899-2904, 2008.

[8] A. V. Lopin, A. V. Semenov, V. M. Puzikov, and A. G. Trushkovsky, "Optical properties of silicon carbide obtained by direct ion deposition," Functional Materials, vol. 13, no. 4, pp. 633-636, 2006

[9] I. A. Salama, N. R. Quick, and A. Kar, "Microstructural and electrical resistance analysis of laser-processed $\mathrm{SiC}$ substrates for wide bandgap semiconductor materials," Journal of Materials Science, vol. 40, no. 15, pp. 3969-3981, 2005.

[10] A. V. Semenov, A. V. Lopin, V. M. Puzikov, V. N. Baumer, and I. N. Dmitruk, "Fabrication of heterostructures based on layered nanocrystalline silicon carbide polytypes," Semiconductors, vol. 44, no. 6, pp. 816-823, 2010.

[11] D. Bimberg, M. Altarelli, and N. O. Lipari, "A calculation of valence band masses, exciton and acceptor energies and the ground state properties of the electron-hole liquid in cubic SiC," Solid State Communications, vol. 40, no. 4, pp. 437-440, 1981.

[12] I. T. Steinberger, E. A. Braun, and E. Alexander, "GuddenPohl and memory effects in an infrared stimulated phosphor," Journal of Physics and Chemistry of Solids, vol. 3, no. 1-2, pp. 133140, 1957.

[13] A. N. Georgobiany and P. A. Pipinys, Tunnel Phenomena in Semiconductors Luminescence, Mir, Moscow, Russia, 1994.

[14] H. Gobrecht and H.-E. Gumlich, "Über den Einfluß der Anregungswellenlänge auf die Elektrophotolumineszenz," Zeitschrift für Physik, vol. 158, no. 2, pp. 226-241, 1960. 

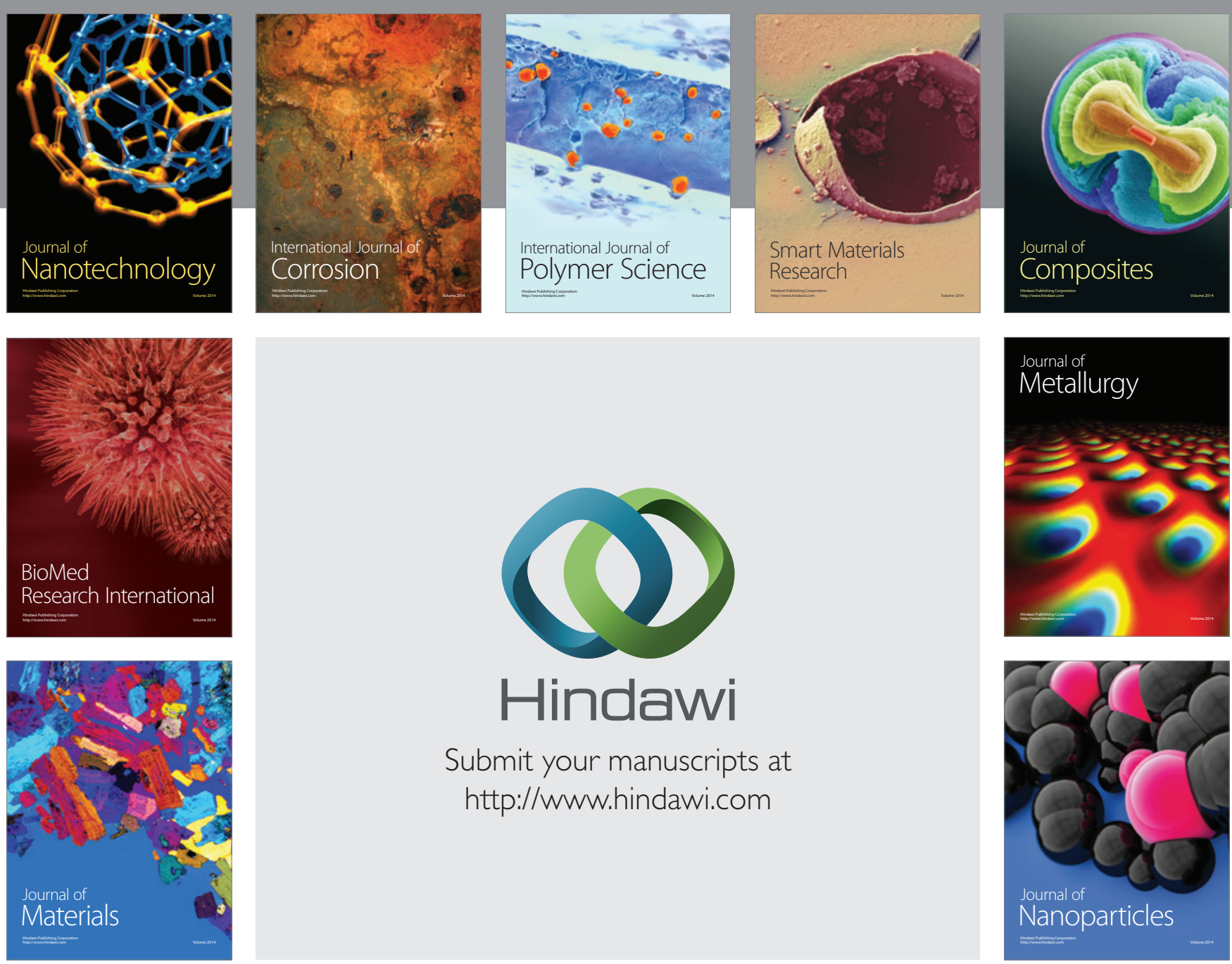

Submit your manuscripts at http://www.hindawi.com
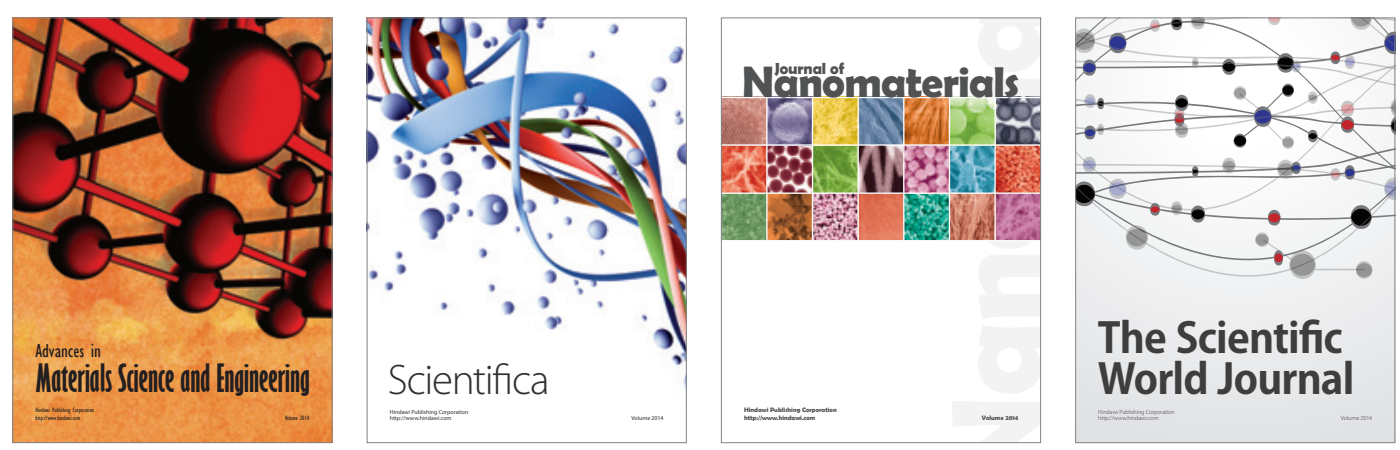

\section{The Scientific World Journal}
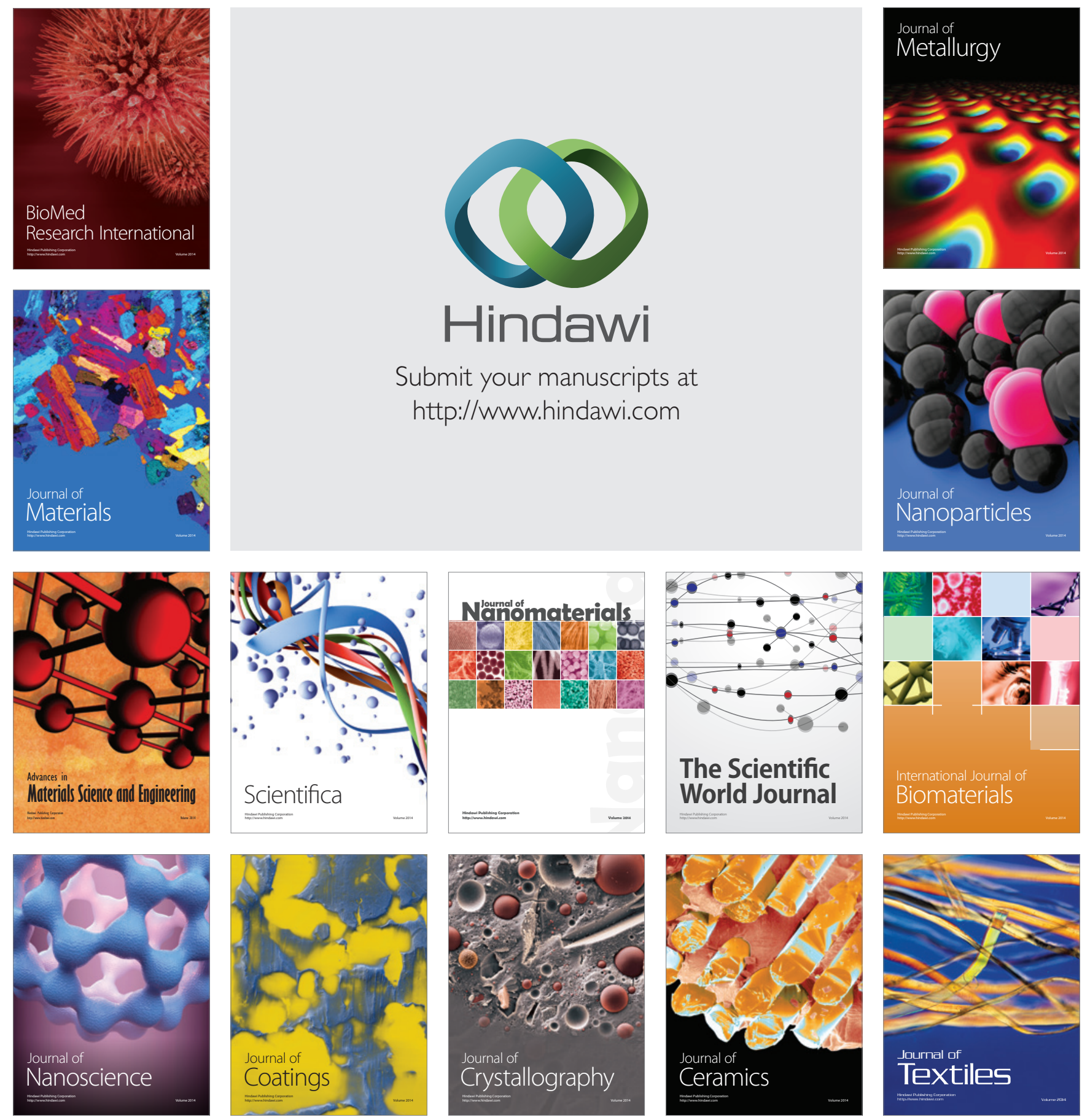